OPEN ACCESS

Edited by:

Fernanda Laezza,

The University of Texas Medical Branch at Galveston, United States

Reviewed by: Boon-Seng Wong, Singapore Institute of Technology,

Singapore

Shannon Risacher, Indiana University Bloomington United States

Fanny M. Elahi, University of California, San Francisco, United States

*Correspondence: Peter J. Snyder pjsnyder@uri.edu

Received: 20 March 2019 Accepted: 07 October 2019 Published: 29 October 2019

Citation:

Cheng DL, Thompson $L$ and Snyder PJ (2019) A Potential Association Between Retinal

Changes, Subjective Memory Impairment, and Anxiety in Older Adults at Risk for Alzheimer's Disease: A 27-Month Pilot Study. Front. Aging Neurosci. 11:288 doi: 10.3389/fnagi.2019.00288

\section{A Potential Association Between Retinal Changes, Subjective Memory Impairment, and Anxiety in Older Adults at Risk for Alzheimer's Disease: A 27-Month Pilot Study}

\author{
Derrick L. Cheng', Louisa Thompson ${ }^{2}$ and Peter J. Snyder ${ }^{2,3 *}$ \\ 1 The Warren Alpert Medical School of Brown University, Providence, Rl, United States, ${ }^{2}$ Department of Neurology, Alpert \\ Medical School, Brown University, Providence, RI, United States, ${ }^{3}$ The Interdisciplinary Neuroscience Program, University \\ of Rhode Island, Kingston, RI, United States
}

Introduction: The utility of subjective memory impairment (SMI) as a risk marker for preclinical Alzheimer's disease (AD) remains unclear; however, recent studies have identified a correlation between retinal biomarkers and onset of preclinical disease. This study examines the relationship between retinal biomarkers that have been associated with cerebral amyloid, an early hallmark of $A D$, and SMI scores in patients at risk for developing AD.

Methods: Forty-nine cognitively normal subjects were followed over 27 months and evaluated using a combination of neuropsychological, psychological, and retinal imaging instruments. Subjective memory testing was conducted using the memory assessment clinic questionnaire (MACQ) and Depression, Anxiety, and Stress Scales (DASS). Multivariate linear analysis was conducted using STATA software.

Results: Positive correlations were found between retinal nerve fiber layer (RNFL) volume and scores obtained from the MAC-Q at 27 months (MAC-Q_27), the DASS questionnaire for anxiety at 27 months (DASS-A_27), and the change in DASS-A over 27 months (dDASSA). There was also a significant positive correlation between these variables and the change in RNFL thickness over 27 months (dRNFL). MACQ_27, DASSA_27, and dDASS-A accounted for $35.7 \%$ of RFNL variance at 27 months and $21.5 \%$ of $\mathrm{dRFNL}$ variance.

Discussion: These findings suggest that worse subjective memory complaints and anxiety scores may be associated with one of the most commonly used structural anatomical retinal markers of early disease burden in $\mathrm{AD}$. If so, these results lend support to SMI as a valid risk marker for later cognitive decline.

Keywords: preclinical Alzheimer's disease, subjective memory impairment, retinal nerve fiber layer, retinal biomarkers, Alzheimer's disease, subjective cognitive decline 


\section{INTRODUCTION}

Alzheimer's disease (AD) is commonly characterized as a neurodegenerative process gradually progressing from mild cognitive impairment to diffuse cognitive impairment and loss of independence in activities of daily living (Sperling et al., 2011). Within the past two decades, research has revealed an earlier "preclinical stage" of $\mathrm{AD}$, often characterized by elevated cerebral $\beta$-Amyloid ( $\mathrm{A} \beta$ ) deposition on positron emission tomography (PET), prior to the development of significant clinical symptoms (Pike et al., 2007). This discovery has propelled a new wave of prevention-based research and clinical drug trials aimed at slowing or halting the onset of AD (Sperling et al., 2011, 2014).

Subjective memory impairment is generally described as self-perceived difficulty with memory or thinking ability and has been associated with depression, anxiety, and prodromal neurodegenerative disease (Abdulrab and Heun, 2008). SMI may be an important feature of preclinical $\mathrm{AD}$ that could help predict a future $\mathrm{AD}$ diagnosis; however, there are several challenges associated with SMI as a risk marker. First, many instruments have been developed that differentially assess SMI, and the field has yet to agree upon its core features or a universal definition (Molinuevo et al., 2017; Rabin et al., 2017). Additionally, SMI often precedes measurable cognitive changes, making it difficult to characterize objectively for research or for evaluating the efficacy of clinical interventions (Molinuevo et al., 2017; Rabin et al., 2017). SMI also co-occurs with anxiety and depression, which have deleterious effects on memory and cognitive function (Green et al., 2003; Modrego and Ferrandez, 2004) and are uniquely associated with increased likelihood of future $\mathrm{AD}$ diagnosis (Yates et al., 2017a,b).

As such, the role of SMI in AD-related cognitive decline is currently under debate. One position is that SMI generally reflects psychiatric overlay such as depression and anxiety and that, as a result, self-reporting is an unreliable metric for predicting cognitive decline (Reisberg and Gauthier, 2008; Reisberg et al., 2008). Conversely, many groups have associated SMI with increased A $\beta$ (Pietrzak et al., 2015), neuroanatomical and metabolic changes (Reisberg and Gauthier, 2008), and decreased cognitive performance (Reisberg and Gauthier, 2008; Koppara et al., 2015).

Comparisons to more well-established AD biomarkers can aid in deciphering what cognitive and subjective symptoms may or may not be associated with AD (Rabin et al., 2015, 2017). Positron emission tomography imaging for neocortical betaamyloid protein aggregation (A $\beta$ PET) is often used as a reference standard against which the predictive power of other potential biomarkers of disease burden or progression may be compared. Because cerebral amyloid deposition begins many years before symptom onset, it can often identify individuals in the preclinical stage of $\mathrm{AD}$. However, PET imaging is expensive, invasive, and not yet widely available (particularly throughout the developing world). As a result, other lower-cost and less invasive imaging modalities are being explored to identify pathological changes associated with preclinical AD.

Current literature suggests that retinal imaging and visual testing may be promising methods for detecting such changes. Pathological findings may include damage to retinal microvasculature (Heringa et al., 2013) as well as retinal nerve fiber layer (RNFL) thinning (Marziani et al., 2013; Cunha et al., 2017a,b; den Haan et al., 2017). Electroretinogram and curcumin binding studies have revealed increased retinal $\mathrm{A} \beta$ and increased latency for the P2 component of visually evoked potentials (VEPs) in AD (Mahajan and Votruba, 2017). A study by Risacher et al. (2013) also identified a potential association between visual contrast sensitivity and $\mathrm{AD}, \mathrm{MCI}$, and subjective complaints. More recently, we have reported moderate correlations between the number and surface area of retinal inclusion bodies that we suspect contain fibrillar $\mathrm{A} \beta$, as well as thinning of the RNFL, and PET cerebral A $\beta$ deposition in preclinical AD (Snyder et al., 2016; Santos et al., 2017).

Our previously reported longitudinal cohort study appears to have been the first to examine potential retinal biomarkers of $\mathrm{AD}$ in cognitively normal individuals with $\mathrm{SMI}$ at increased risk for AD (Snyder et al., 2016; Santos et al., 2017). Here, using the same data repository, we have explored the potential associations between SMI, self-reported mood and anxiety symptoms, and RNFL volumes as a first step toward characterizing the relationship between these variables and how they may relate to the potential for developing $\mathrm{AD}$.

\section{MATERIALS AND METHODS}

Sixty-three older adults with elevated risk of developing $\mathrm{AD}$ (determined by first-degree family history of $\mathrm{AD}$ and apolipoprotein E4 (ApoE4) gene expression) were followed for 27 months (Table 1). These criteria (i.e., family history, SMI, and E4 carrier status) were chosen based on the established literature demonstrating their combined association with a $60 \%$ likelihood of concurrent cerebral amyloidosis and increased risk for the development of AD (Mielke, 2012). Moreover, these criteria have been used to define preclinical AD cohorts in several prior studies (Kulic and Unschuld, 2016; Snyder et al., 2016; Golzan et al., 2017; Ott et al., 2017). In accordance with the guidelines established by the subjective cognitive decline initiative (SCD-I) working group, the trial was limited to cognitively normal subjects and excluded subjects with mini-mental status exam (MMSE) scores of under 28 (Jessen et al., 2014). Participants were also screened for cognitive impairment using a telephonic cognitive screening test - the minnesota cognitive acuity screen (MCAS) (Tremont et al., 2011). Subjects with a diagnosis of MCI or AD and history of ophthalmologic disease such as age-related macular degeneration (AMD) or cataracts were excluded.

Subjects completed the 6-item memory assessment clinic questionnaire (MAC-Q) (Crook et al., 1992), 42-item Depression, Anxiety, and Stress Scale (DASS) (Crawford and Henry, 2003), mini-mental status examination (MMSE) (Folstein et al., 1975), and retinal spectral-domain optical coherence tomography study (SD-OCT; SPECTRALIS, Heidelberg Engineering) at baseline and 27 months later. Fourteen subjects were either unable to return for their 27-month end-of-study visits or were unable to complete the retinal imaging component of the study (final sample, $N=49,57 \%$ female). Using SD-OCT, RNFL volumes 
TABLE 1 | Participant demographic information and baseline, 27-month, and change scores values for self-report variables and RNFL volumes.

\begin{tabular}{|c|c|c|c|}
\hline Variable name Variable name & Mean & $S D$ & Min/Max \\
\hline Age (years) & 63.06 & 5.42 & 53,75 \\
\hline Education (years) & 17.21 & 2.77 & 12,24 \\
\hline \multicolumn{4}{|l|}{ Sex } \\
\hline Female & $57.14 \%$ & & \\
\hline Male & $42.86 \%$ & & \\
\hline \multicolumn{4}{|l|}{ ApoE genotype } \\
\hline$\varepsilon 2 / \varepsilon 3$ & $12.22 \%$ & & \\
\hline$\varepsilon 3 / \varepsilon 3$ & $42.86 \%$ & & \\
\hline$\varepsilon 3 / \varepsilon 4$ & $38.78 \%$ & & \\
\hline$\varepsilon 4 / \varepsilon 4$ & $6.122 \%$ & & \\
\hline ApoE4 gene dose & 0.51 & 0.62 & 0,2 \\
\hline MMSE (baseline) & 29.00 & 1.02 & 27,30 \\
\hline MMSE (27 months) & 29.18 & 1.34 & 26,30 \\
\hline \multicolumn{4}{|l|}{ Baseline values } \\
\hline MAC-Q & 26.12 & 3.27 & 19, 33 \\
\hline DASS-D & 2.73 & 4.63 & 0,22 \\
\hline DASS-A & 2.16 & 3.08 & 0,12 \\
\hline DASS-S & 5.92 & 4.70 & 0,18 \\
\hline RNFL & 0.23 & 0.02 & $0.19,0.28$ \\
\hline \multicolumn{4}{|l|}{ 27-month values } \\
\hline MAC-Q & 21.61 & 2.64 & 16,28 \\
\hline DASS-D & 2.65 & 4.53 & 0,26 \\
\hline DASS-A & 2.02 & 3.14 & 0,18 \\
\hline DASS-S & 4.73 & 4.18 & 0,14 \\
\hline RNFL & 0.21 & 0.02 & $0.17,0.30$ \\
\hline \multicolumn{4}{|l|}{ Change values } \\
\hline MAC-Q & -4.51 & 2.93 & $-10,3$ \\
\hline DASS-D & -0.08 & 4.51 & $-17,17$ \\
\hline DASS-A & -0.14 & 2.87 & $-8,11$ \\
\hline DASS-S & -1.18 & 3.92 & $-10,9$ \\
\hline RNFL & -0.02 & 0.02 & $-0.05,0.04$ \\
\hline
\end{tabular}

were obtained within the macular region extending $3.45 \mathrm{~mm}$ from the foveal center. For each subject, OD and OS values were averaged and mean values were calculated; values for individual eyes were not recorded. Change scores over the 27-month period were obtained for each measure.

Two separate multivariate linear regression models were conducted using STATA (StataCorp College Station, TX, United States), one using RNFL volume at 27 months (RNFL_27), and the other using change in RNFL over 27 months (dRNFL) as dependent variables. Change values were obtained by subtracting baseline RNFL from RNFL at 27 months such that larger dRNFL values signified reduced volume due to RNFL thinning. Independent variables were 27-month MAC-Q scores (MACQ_27), change in MAC-Q scores over 27 months (dMACQ), 27-month self-reported depression, anxiety, and stress scales (DASSD_27, DASSA_27, DASSS_27, respectively), and change in self-reported depression, anxiety, and stress scales over 27 months (dDASSD, dDASSA, and dDASSS, respectively). Change values were obtained for each measure. Co-linearity was assessed using a statistics package in Microsoft Excel. After assessing for co-linearity between age, sex, genetic load, and the previously described independent variables, an additional multivariate regression was conducted to assess the effect of the independent variables with and without age, sex, and other co-variates, respectively, on the dependent variable. Further statistical analysis was conducted on MACQ_27, DASSA_27, and dDASSA due to significant correlation.

This study was approved by and complied with the regulations of the Rhode Island Hospital Institutional Review Board (Lifespan Hospital Systems, Providence, RI, United States). All participants gave written consent in accordance with the Declaration of Helsinki. This study complied with HIPAA regulations.

\section{RESULTS}

An overview of sample results for average baseline, 27-month, and delta values for MAC-Q, DASS, and RNFL testing can be seen below in Table 1. At 27 months, correlations were found between RNFL_27 and MACQ_27 (Adj $\left.R^{2}=0.16, p<0.01\right)$, DASSA_27 (Adj $R^{2}=0.13, p<0.01$ ), and dDASSA (Adj $R^{2}=0.25, p<0.01$ ), such that reduced RNFL volume at 27 months was associated with increased memory and anxiety complaints at 27 months. The relationships between 27-month values for RNFL_27 and DASSD_27, DASSS_27, dMACQ, dDASSD, and dDASSS were not significant.

There was also a significant positive correlation between dRFNL and MACQ_27 (Adj $\left.R^{2}=0.06, p=0.05\right)$, DASSA_27 (Adj $R^{2}=0.10, p=0.02$ ), and dDASSA (Adj $R^{2}=0.11, p=0.01$ ) such that RNFL volume loss over 27 months was associated with increased memory and anxiety complaints at 27 months. There were no significant correlations between dRNFL and DASSD_27 $(p=0.75)$, DASSS_27 $(p=0.52)$, dMACQ $(p=0.28)$, dDASSD $(p=0.54)$, or dDASSS $(p=0.78)$.

Individual regression models can be seen in Figures 1-6 below. These individual regression models identify possible outliers on DASSA_27 and dDASSA testing. When removing one outlier whose DASSA_27 score was 18 (compared to average DASSA_27 2.02), the correlation between DASSA_27 and RNFL and between DASSA_27 and dRNFL is not significant ( $p=0.96$ and $p=0.93$, respectively). The correlation between dDASSA and RNFL and between dDASSA and dRNFL remains significant ( $p=0.05, p=0.03$, respectively) after removal of one significant outlier. Importantly, there were no statistically significant correlations between either DASSA_27 or MACQ_27 with MMSE scores at 27 months or with change in MMSE scores over 27 months $(p>0.05)$. Assessment of co-linearity demonstrated mild to moderate correlations between DASS-S, DASS-D, DASS-A, and MAC-Q scores ranging from 0.07 to 0.46 suggesting that these variables are separate but related constructs; of these, only correlations between DASS-D and DASS-S, DASS$A$ and DASS-S, and DASS-A and MAC-Q were significant ( $p=0.01, p=0.01, \mathrm{p}<0.01$, respectively). All other correlations between anxiety, depression, stress, and cognitive scores were not significant $(p>0.05)$.

Further multivariate regression models were conducted on statistically relevant variables MACQ_27, DASSA_27, and 


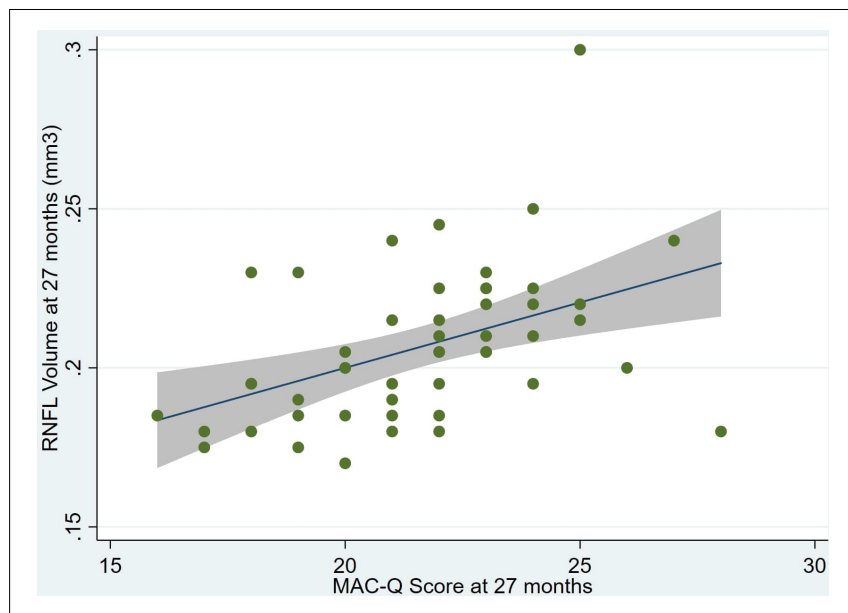

FIGURE 1 | Individual regression models for MACQ_27 using baseline RNFL volumes (RNFL) as the dependent variable.

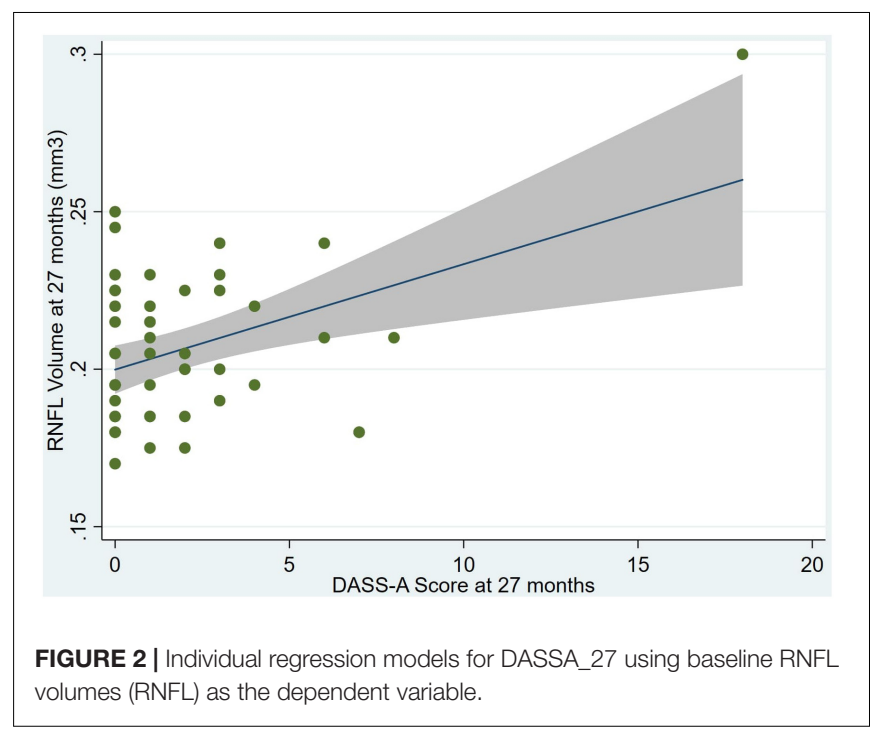

dDASSA. Statistical results from the multivariate models can be seen in Tables 2, 3. Together, MACQ_27, DASSA_27, and DDASSA accounted for approximately $35.7 \%$ of RNFL_27 variance (Adj. $R^{2}=0.36 ; p<0.01$ ) and approximately $21.5 \%$ of dRFNL variance (Adj. $R^{2}=0.22 ; p<0.01$ ). For RFNL_27, the effects of MACQ_27 and dDASSA were significant $(p=0.02$, $p<0.01)$ while DASSA_27 was not $(p=0.35)$. For dRFNL, the effect of MACQ_27 was significant $(p=0.02)$, while DASSA_27 and dDASS-A were not ( $p=0.11$ and $p=0.25$, respectively). When accounting for age, sex, or APOE4 status, there was no significant change in variance of RNFL_27 or dRNFL.

\section{DISCUSSION}

Recent studies suggest that RNFL thinning is potentially associated with increased cerebral amyloid deposition in the preclinical stage of $\mathrm{AD}$ and may have potential as a surrogate

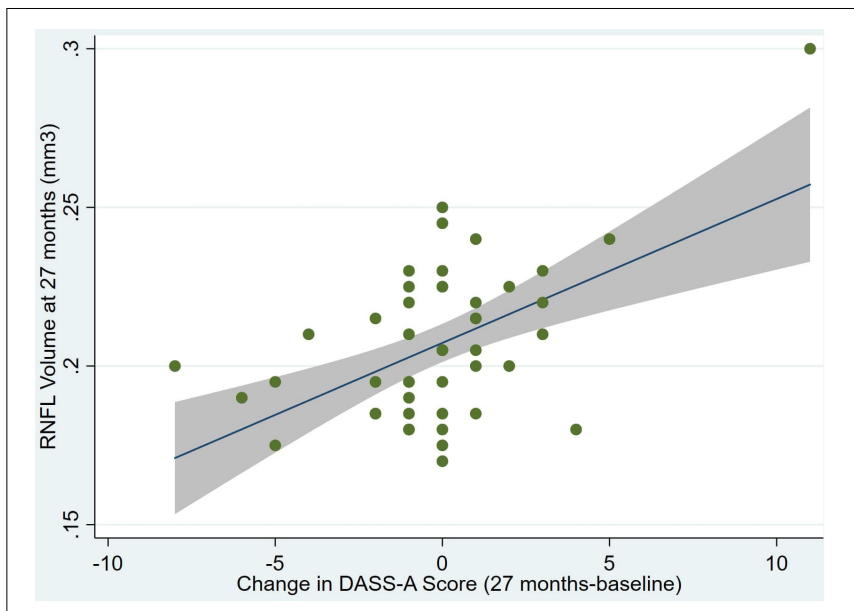

FIGURE 3 | Individual regression models for dDASSA using baseline RNFL volumes (RNFL) as the dependent variable.

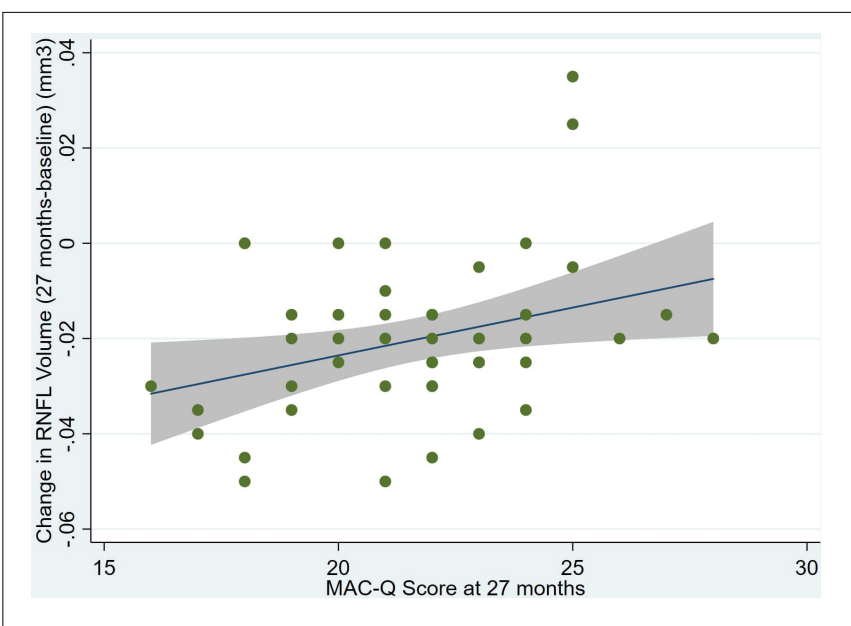

FIGURE 4 | Individual regression models for MACQ_27 using change in RNFL volume (dRNFL) as the dependent variable.

biomarker for $\mathrm{AD}$, if sufficiently validated through further research. The goal of this study was to assess whether subjective cognitive and psychiatric complaints that have also been associated with the early stages $\mathrm{AD}$ are correlated with lower RNFL volumes due to thinning of the RNFL over time.

In this study, we followed 49 cognitively normal older adults with increased AD risk for 27 months using SD-OCT, MMSE, MAC-Q, and DASS. We found that both worse reported anxiety at 27 months, as well as increases in self-reported symptoms of anxiety over 27 months, were associated with reduced RNFL volume at 27 months. Similarly, worse SMI scores at 27 months were associated with reduced RNFL volume and increased RNFL thinning. These data suggest that SMI and self-reported anxiety are associated with RNFL volume and RNFL thinning, two retinal biomarkers previously shown to correlate with cerebral $\mathrm{A} \beta$ deposition.

This is an initial report with a relatively small sample size. Future work may indicate whether elevated DASS-A scores reflect 


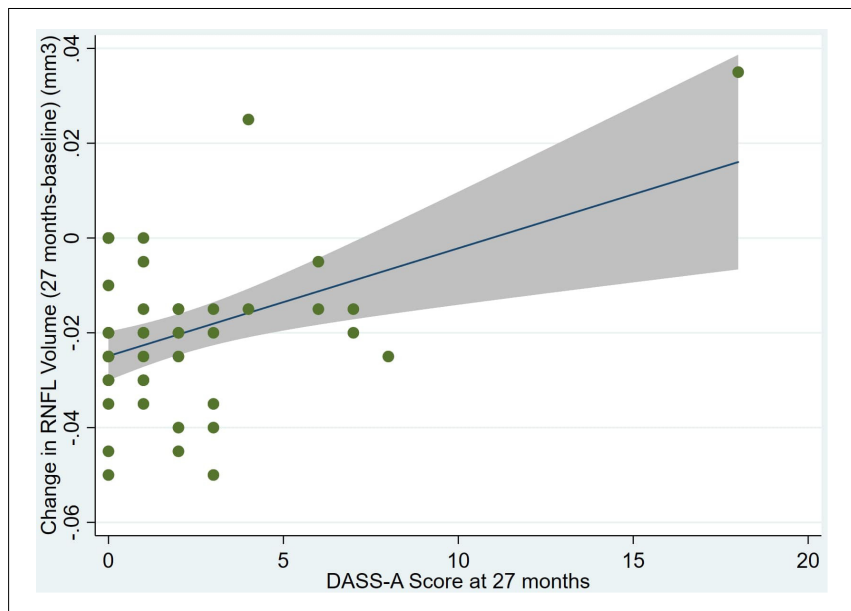

FIGURE 5 | Individual regression models for DASSA_27 using change in RNFL volume (dRNFL) as the dependent variable.

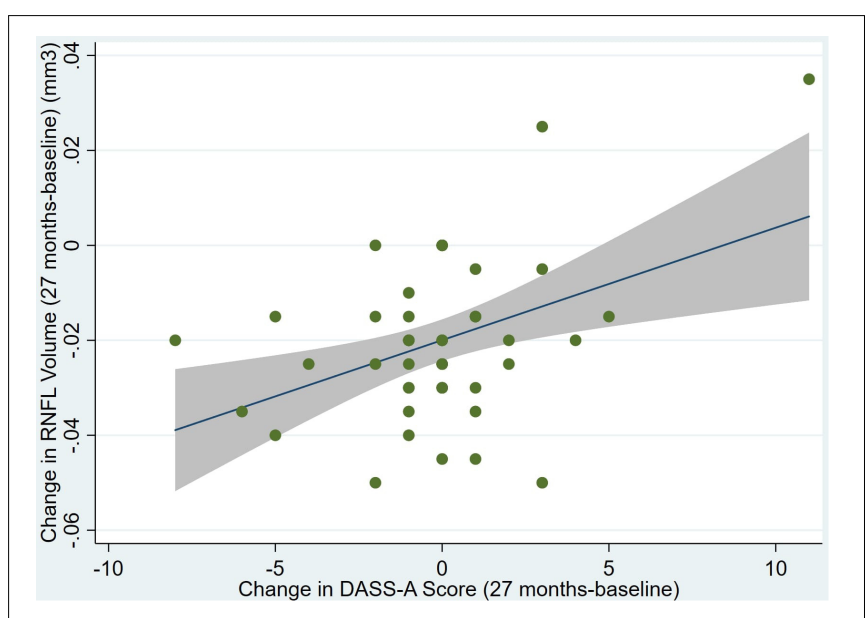

FIGURE 6 | Individual regression models for dDASSA using change in RNFL volume (dRNFL) as the dependent variable.

anxiety as a direct result of SMI, an association that is challenging to evaluate with self-report questionnaires. Notably, baseline values for RNFL and MAC-Q, DASS-D, DASS-A, and DASS-S demonstrated positive correlations with each other, but were not statistically significant, and hence effect magnification over time may have contributed to our findings, to a degree.

Interestingly, unlike anxiety, baseline, delta, and 27-month scores for self-reported stress and depression were not associated with RNFL volume or changes in RNFL volume. This finding may be consistent with prior research suggesting that anxiety is uniquely predictive of cognitive decline and AD (Teri et al., 1999; Sinoff and Werner, 2003; Balash et al., 2013). For example, Pietrzak et al. (2015) found that high anxiety, $A \beta+$ older adults demonstrated a more precipitous cognitive decline compared to those with low anxiety or depression alone. These findings suggest that self-reported anxiety may play a significant role in preclinical $\mathrm{AD}$ and future cognitive decline, even when compared to measures of depression or stress.
TABLE 2 | Statistical results from multivariate model with RNFL_27 as a dependent variable.

\begin{tabular}{|c|c|c|c|c|c|c|}
\hline & Coefficient & SE & $T$-Score & $P$-Value & $\begin{array}{c}\text { 95\%Cl: } \\
\text { Low }\end{array}$ & $\begin{array}{c}\text { 95\%Cl: } \\
\text { High }\end{array}$ \\
\hline MACQ_27 & 0.15 & 0.02 & 5.93 & $4 \mathrm{E}-07$ & 0.10 & 0.20 \\
\hline DASSA_27 & $1.02 \mathrm{E}-3$ & $1.08 \mathrm{E}-3$ & 0.95 & 0.35 & $-1.14 \mathrm{E}-3$ & $3.19 \mathrm{E}-3$ \\
\hline dDASSA & $2.70 E-3$ & $1.16 \mathrm{E}-3$ & 2.32 & 0.02 & $0.36 E-3$ & $5.04 \mathrm{E}-3$ \\
\hline
\end{tabular}

TABLE 3 | Statistical results from multivariate model with dRNFL as a dependent variable.

\begin{tabular}{lcccccc}
\hline & Coefficient & SE & T-Score & $\boldsymbol{P}$-Value & $\begin{array}{c}\mathbf{9 5 \% C l :} \\
\text { Low }\end{array}$ & $\begin{array}{c}\mathbf{9 5 \% C l :} \\
\text { High }\end{array}$ \\
\hline MACQ_27 & -0.04 & 0.02 & -2.41 & 0.02 & -0.08 & -0.01 \\
DASSA_27 & $1.33 E-3$ & $0.80 E-3$ & 1.65 & 0.11 & $-0.29 \mathrm{E}-3$ & $2.94 \mathrm{E}-3$ \\
dDASSA & $1.00 \mathrm{E}-3$ & $0.87 \mathrm{E}-3$ & 1.16 & 0.25 & $-0.74 \mathrm{E}-3$ & $2.75 \mathrm{E}-3$ \\
\hline
\end{tabular}

A common concern noted, with respect to reliance on SMI as a risk marker for preclinical $\mathrm{AD}$, is the confounding factor of true cognitive decline (CD). In order to control for this in the current study, we required an MMSE score of $\geq 28$. We did not find any significant change in MMSE over the 27-month period, and we likewise did not find any correlation between MMSE and MAC-Q/DASS. This supports the hypothesis that $\mathrm{SMI}$ may play a role in preclinical $\mathrm{AD}$ independent from objective cognitive impairment.

However, there are several limitations to this study. First, our analysis was limited to 49 subjects followed over the span of 27 months. Replication in a larger cohort followed over a longer span of time will be necessary to establish the strength and generalizability of these findings. The study cohort was limited to cognitively normal subjects characterized as high-risk for developing $\mathrm{AD}$ based on first-degree family history, ApoE4 carrier status, and SMI, but the presence of biomarker-defined (i.e., $\mathrm{A} \beta$ PET) preclinical $\mathrm{AD}$ could not be confirmed, which limits the interpretation of our findings to a degree. Future studies associating SMI and retinal biomarkers to established AD biomarkers (e.g., tau and amyloid) in CSF or PET imaging data are therefore an important next step for this line or research. Moreover, our high-AD risk sample characterizes only one subset of individuals who may develop $\mathrm{AD}$, and our findings may not extend to the broader older adult population characterized by more variable levels of $\mathrm{AD}$ risk. Future studies would ideally include comparison groups of ApoE4 positive subjects with clinical disease as well as cognitively normal subjects without risk factors for $\mathrm{AD}$ in order to clarify the generalizability of these findings. Although we did not observe any significant effects of age, sex, or APOE status in our analyses, these variables could still emerge as important moderators with a larger sample. Finally, recent studies have suggested that retinal thinning in AD may be more pronounced in the superior hemiretina compared to the perimacular retina; therefore, further research may benefit from imaging larger retinal areas (den Haan et al., 2017). Overall, additional research is needed to provide further validation as RNFL as an AD biomarker. 
Despite these limitations, we consider this current report to be "path finding" in suggesting a novel relationship between subjective memory impairment (SMI), anxiety, and RNFL changes in older adults at high risk for AD. As the field of research in preclinical $\mathrm{AD}$ continues to expand, future studies may further validate these findings. Though there are inherent challenges with the use of SMI in both research and clinical practice, we suggest that SMI may potentially be a reasonable adjunct to objective biomarkers of preclinical $\mathrm{AD}$, especially when examined in concordance with literature-established guidelines.

\section{CONCLUSION}

The role of SMI in preclinical $\mathrm{AD}$ has been the subject of continuing debate in the literature. In this study we found a significant correlation between SMI (as measured by the memory assessment questionnaire and depression, anxiety, and stress scales) and RNFL volume- metrics that have previously been implicated in preclinical AD (Lu et al., 2010; Snyder et al., 2016; Santos et al., 2017; Ko et al., 2018; Sanchez et al., 2018). This supports the potential use of SMI as a risk marker for preclinical disease and its incorporation into the developing array of preclinical $\mathrm{AD}$ assessment tools. Further validation of SMI with other preclinical assessment tools such as PET imaging, macular pigment optical density (MPOD), and retinal vasculature imaging (e.g., measurement of vascular bed complexity and tortuosity) is needed. Despite these challenges, our findings suggest that SMI should be considered as a reasonable risk marker, to augment other more objective methods, of screening for preclinical AD.

\section{REFERENCES}

Abdulrab, K., and Heun, R. (2008). Subjective memory impairment. a review of its definitions indicates the need for a comprehensive set of standardised and validated criteria. Eur. Psychiatry 23, 321-330. doi: 10.1016/j.eurpsy.2008. 02.004

Balash, Y., Mordechovich, M., Shabtai, H., Giladi, N., Gurevich, T., and Korczyn, A. D. (2013). Subjective memory complaints in elders: depression, anxiety, or cognitive decline? Acta Neurol. Scand. 127, 344-350. doi: 10.1111/ane. 12038

Crawford, J. R., and Henry, J. D. (2003). The depression anxiety stress scales (DASS): normative data and latent structure in a large non-clinical sample. J. Clin. Psychol. 42, 111-131. doi: 10.1348/014466503321 903544

Crook, T. H. III, Feher, E. P., and Larrabee, G. J. (1992). Assessment of memory complaint in age-associated memory impairment: the MAC-Q. Int. Psychogeriatr. 4, 165-176. doi: 10.1017/s1041610292000991

Cunha, J. P., Proenca, R., Dias-Santos, A., Almeida, R., Aguas, H., Alves, M., et al. (2017a). OCT in Alzheimer's disease: thinning of the RNFL and superior hemiretina. Graefes Arch. Clin. Exp. Ophthalmol. 255, 1827-1835. doi: 10.1007/ s00417-017-3715-9

Cunha, J. P., Proenca, R., Dias-Santos, A., Melancia, D., Almeida, R., Aguas, H., et al. (2017b). Choroidal thinning: Alzheimer's disease and aging. Alzheimers Dement. 8, 11-17.

den Haan, J., Verbraak, F. D., Visser, P. J., and Bouwman, F. H. (2017). Retinal thickness in Alzheimer's disease: a systematic review and metaanalysis. Alzheimers Dement. 6, 162-170. doi: 10.1016/j.dadm.2016. 12.014

\section{DATA AVAILABILITY STATEMENT}

The datasets for this manuscript are not publicly available because they include protected health information. The datasets generated for this study are available on request to corresponding author.

\section{ETHICS STATEMENT}

This study was approved by and complied with the regulations of the Rhode Island Hospital Institutional Review Board (Lifespan Hospital Systems, Providence, RI, United States). All participants gave written consent in accordance with the Declaration of Helsinki. This study complied with HIPAA regulations.

\section{AUTHOR CONTRIBUTIONS}

DC and LT contributed to the data gathering and analysis, and manuscript preparation and revision. PS contributed to the study design, data gathering and analysis, and final manuscript preparation.

\section{FUNDING}

The authors declare that this study received funding from Pfizer Inc., in the form of an unrestricted research grant. The funder was not involved in the study design, collection, analyses, interpretation of data, the writing of this article, nor the decision to submit it for publication.

Folstein, M. F., Folstein, S. E., and McHugh, P. R. (1975). Mini-mental state: a practical method for grading the cognitive state of patients for the clinician. J. Psych. Res. 12, 189-198.

Golzan, S. M., Goozee, K., Georgevsky, D., Avolio, A., Chatterjee, P., Shen, K., et al. (2017). Retinal vascular and structural changes are associated with amyloid burden in the elderly: ophthalmic biomarkers of preclinical Alzheimer's disease. Alzheimers Res. Ther. 9:13. doi: 10.1186/s13195-017-0239-9

Green, R. C., Cupples, L. A., Kurz, A., Auerbach, S., Go, R., Sadovnick, D., et al. (2003). Depression as a risk factor for Alzheimer Disease. Arch. Neurol. 60, 753-759.

Heringa, S. M., Bouvy, W. H., van den Berg, E., Moll, A. C., Kappelle, L. J., and Biessels, G. J. (2013). Associations between retinal microvascular changes and dementia, cognitive functioning, and brain imaging abnormalities: a systematic review. J. Cereb. Blood Flow Metab. 33, 983-995. doi: 10.1038/jcbfm.2013.58

Jessen, F., Amariglio, R. E., van Boxtel, M., Breteler, M., Ceccaldi, M., Chetelat, G., et al. (2014). A conceptual framework for research on subjective cognitive decline in preclinical Alzheimer's disease. Alzheimers Dement. 10, 844-852. doi: 10.1016/j.jalz.2014.01.001

Ko, F., Muthy, Z. A., Gallacher, J., Sudlow, C., Rees, G., Yang, Q., et al. (2018). Association of retinal nerve fiber layer thinning with current and future cognitive declinea study using optical coherence tomography. JAMA Neurobiol. 75, 1198-1205. doi: 10.1001/jamaneurol.2018.1578

Koppara, A., Wagner, M., Lange, C., Ernst, A., Wiese, B., Konig, H. H., et al. (2015). Cognitive performance before and after the onset of subjective cognitive decline in old age. Alzheimers Dement. 1, 194-205. doi: 10.1016/j.dadm.2015.02.005

Kulic, L., and Unschuld, P. G. (2016). Recent advances in cerebrospinal fluid biomarkers for the detection of preclinical Alzheimer's disease. Curr. Opin. Neurol. 29, 749-755. doi: 10.1097/wco.0000000000000399 
Lu, Y., Li, Z., Zhang, X., Ming, B., Jia, J., Wang, R., et al. (2010). Retinal nerve fiber layer structure abnormalities in early Alzheimer's disease: evidence in optical coherence tomography. Neurosci. Lett. 480, 69-72. doi: 10.1016/j.neulet.2010. 06.006

Mahajan, D., and Votruba, M. (2017). Can the retina be used to diagnose and plot the progression of Alzheimer's disease? Acta Ophthalmol. 95, 768-777. doi: 10.1111 /aos.13472

Marziani, E., Pomati, S., Ramolfo, P., Cigada, M., Giani, A., Mariani, C., et al. (2013). Evaluation of retinal nerve fiber layer and ganglion cell layer thickness in Alzheimer's disease using spectral-domain optical coherence tomography. Invest. Ophthalmol. Vis. Sci. 54, 5953-5958. doi: 10.1167/iovs.13-12046

Mielke, M. M. (2012). Indicators of amyloid burden in a population-based study of cognitively normal elderly. Neurology 79, 1570-1577. doi: 10.1212/WNL. 0b013e31826e 2696

Modrego, P. J., and Ferrandez, J. (2004). Depression in ptaients with mild cognitive impairment increases the risk of developing dementia of Alzheimer type. Arch. Neurol. 61, 1290-1293.

Molinuevo, J. L., Rabin, L. A., Amariglio, R., Buckley, R., Dubois, B., Ellis, K. A., et al. (2017). Implementation of subjective cognitive decline criteria in research studies. Alzheimers Dement. 13, 296-311. doi: 10.1016/j.jalz.2016. 09.012

Ott, B. R., Jones, R. N., Noto, R. B., Yoo, D. C., Snyder, P. J., Bernier, J. N., et al. (2017). Brain amyloid in preclinical Alzheimer's disease is associated with increased driving risk. Alzheimers Dement. 6, 136-142. doi: 10.1016/j.dadm. 2016.10.008

Pietrzak, R. H., Lim, Y. Y., Neumeister, A., Ames, D., Ellis, K. A., Harrington, K., et al. (2015). Amyloid-beta, anxiety, and cognitive decline in preclinical Alzheimer disease: a multicenter, prospective cohort study. JAMA Psychiatry 72, 284-291. doi: 10.1001/jamapsychiatry.2014.2476

Pike, K. E., Savage, G., Villemagne, V. L., Ng, S., Moss, S. A., Maruff, P., et al. (2007). Beta-amyloid imaging and memory in non-demented individuals: evidence for preclinical Alzheimer's disease. Brain 130(Pt 11), 2837-2844. doi: 10.1093/ brain/awm 238

Rabin, L. A., Smart, C. M., and Amariglio, R. E. (2017). Subjective cognitive decline in preclinical Alzheimer's Disease. Annu. Rev. Clin. Psychol. 13, 369-396. doi: 10.1146/annurev-clinpsy-032816-045136

Rabin, L. A., Smart, C. M., Crane, P. K., Amariglio, R. E., Berman, L. M., Boada, M., et al. (2015). Subjective cognitive decline in older adults: an overview of self-report measures used across 19 international research studies. J. Alzheimers Dis. 48(Suppl. 1), S63-S86. doi: 10.3233/JAD-150154

Reisberg, B., and Gauthier, S. (2008). Current evidence for subjective cognitive impairment (SCI) as the pre-mild cognitive impairment (MCI) stage of subsequently manifest Alzheimer's disease. Int. Psychogeriatr. 20, 1-16. doi: 10.1017/s1041610207006412

Reisberg, B., Prichep, L., Mosconi, L., John, E. R., Glodzik-Sobanska, L., Boksay, I., et al. (2008). The pre-mild cognitive impairment, subjective cognitive impairment stage of Alzheimer's disease. Alzheimers Dement. 4(1 Suppl. 1), S98-S108. doi: 10.1016/j.jalz.2007.11.017
Risacher, S. L., Wudunn, D., Pepin, S. M., MaGee, T. R., McDonald, B. C., Flashman, L. A., et al. (2013). Visual contrast sensitivity in Alzheimer's disease, mild cognitive impairment, and older adults with cognitive complaints. Neurobiol. Aging 34, 1133-1144. doi: 10.1016/j.neurobiolaging.2012.08.007

Sanchez, D., Castilla-Marti, M., Rodriguez-Gomez, O., Valero, S., Piferrer, A., Martinez, G., et al. (2018). Usefulness of peripapillary nerve fiber layer thickness assessed by optical coherence tomography as a biomarker for Alzheimer's disease. Sci. Rep. 8:16345.

Santos, C. Y., Johnson, L. N., Lim, Y. Y., Fernandez, B. M., Ott, B. R., Salloway, S., et al. (2017). Retinal nerve fiber layer and ganglion cell layer volume changes in preclinical Alzheimer's Disease over 27 months. Alzheimers Dement. 13:1280.

Sinoff, G., and Werner, P. (2003). Anxiety disorder and accompanying subjective memory loss in the elderly as a predictor of future cognitive decline. Int. J. Geriatr. Psychiatry 18, 951-959. doi: 10.1002/gps.1004

Snyder, P. J., Johnson, L. N., Lim, Y. Y., Santos, C. Y., Alber, J., Maruff, P., et al. (2016). Nonvascular retinal imaging markers of preclinical Alzheimer"s disease. Alzheimers Dement. 4, 169-178. doi: 10.1016/j.dadm.2016.09.001

Sperling, R., Mormino, E., and Johnson, K. (2014). The evolution of preclinical Alzheimer"s disease: implications for prevention trials. Neuron 84, 608-622. doi: 10.1016/j.neuron.2014.10.038

Sperling, R. A., Aisen, P. S., Beckett, L. A., Bennett, D. A., Craft, S., Fagan, A. M., et al. (2011). Toward defining the preclinical stages of Alzheimer's disease: recommendations from the National Institute on Aging-Alzheimer's Association workgroups on diagnostic guidelines for Alzheimer's disease. Alzheimers Dement. 7, 280-292. doi: 10.1016/j.jalz.2011.03.003

Teri, L., Ferretti, L. E., and Gibbons, L. E. (1999). Anxiety in Alzheimer's Disease: prevalence and comorbidity. J. Gerontol. 54, 348-352.

Tremont, G., Papandonatos, G. D., Springate, B., Huminski, B., McQuiggan, M. D., Grace, J., et al. (2011). Use of the telephone-administered minnesota cognitive acuity screen to detect mild cognitive impairment. Am. J. Alzheimers Dis. Other Demen. 26, 555-562. doi: 10.1177/1533317511428151

Yates, J. A., Clare, L., Woods, R. T., Cognitive Function Ageing Study Wales, (2017a). What is the relationship between health, mood, and mild cognitive impairment? J. Alzheimers Dis. 55, 1183-1193. doi: 10.3233/jad-160611

Yates, J. A., Clare, L., Woods, R. T., and Mrc, C. (2017b). Subjective memory complaints, mood and MCI: a follow-up study. Aging Ment. Health 21, 313-321. doi: $10.1080 / 13607863.2015 .1081150$

Conflict of Interest: The authors declare that the research was conducted in the absence of any commercial or financial relationships that could be construed as a potential conflict of interest.

Copyright (c) 2019 Cheng, Thompson and Snyder. This is an open-access article distributed under the terms of the Creative Commons Attribution License (CC BY). The use, distribution or reproduction in other forums is permitted, provided the original author(s) and the copyright owner(s) are credited and that the original publication in this journal is cited, in accordance with accepted academic practice. No use, distribution or reproduction is permitted which does not comply with these terms. 